\title{
Improving Hospital Services using Cloud and Speech to Text Technologies
}

\author{
Florin Daniel Anton, Silviu Raileanu, Silvia Anton, Nick Ivanescu
}

\begin{abstract}
Nowadays in many hospitals all over the world there is an acute lack of physicians. In many cases in such hospitals, the physicians are overwhelmed by a large number of patients which they must consult or visit after a surgery. In order to help them, the physicians are accompanied by resident students which are learning from the physician, but also must note his observations, usually there is a student in the group which will note the observations and then, after the visit he will wrote down on the patient observation sheet the physician observations. Many times, the student is not able to write everything down and he is writing what he is remembering resulting that important information will be omitted. To solve this problem, the paper proposes an information system which allow the physician to record his observations using a smart phone, then the recording is converted to text using a cloud speech to text service and added to the patient observation sheet. The system allows to preserve the confidentiality of medical data and addition of new (medical) words to dictionary.
\end{abstract}

Keywords - cloud services, hospital information system, medical record confidentiality, patient electronic observation sheet, speech to text

\section{Introduction}

Each patient in the hospital has an observation sheet, this is an important tool which can be used by the physician to monitor the evolution of the patient and the response to the medication. Depending on the hospital and the specialty, the observation sheet may vary al the level of the information which is included but there are also common fields [1], [2].

The observation sheet is analyzed by the physician, and sometimes when the case is complicated, or the physician doesn't have the expertise, an opinion from an expert is requested and in this case the patient observation sheet is available also to the expert in order to see the medical history of the patient. If the observation sheet is available only in hard copy this process will be cumbered. A solution for this problem is to use an electronic observation sheet, solution which has been implemented in many cases. Using an electronic observation sheet allows that medical data to be retrieved much easier and faster [3], [4], allows that the analysis of the data can be done internally or outside the

Florin Daniel Anton, Silviu Raileanu, Silvia Anton, Nick Ivanescu University Politehnica of Bucharest Romania

This work was supported by a grant of the Romanian National Authority for Scientific Research and Innovation, CNCS/CCCDI UEFISCDI, project number PN-III-P2-2.1-PED-2016-1336, within PNCDI III. hospital [5], [6], and it improves the quality of the medical service [7]. When storing the patient information in electronic format there are also some disadvantages or question marks related to privacy [8].

The privacy of the electronic medical record has been approached in different papers in the scientific literature and is a problem which is still debated; in [9] a literature review has been done regarding two important topics: security and the privacy of the electronic health record (EHR) systems. An important problem is related to the way that data is gathered and published from EHR, in [10] a survey related to the algorithms proposed to process and publish patient data which are also preserving the privacy has been conducted. A similar problem regarding the access control and sharing patient medical data was also discussed in [11].

Privacy of medical data is a very sensitive topic approached by a series of papers, in [12] is presented a framework which proposes the usage of Statistical Disclosure Control (SDC) methods on non-numerical clinical data, preserving the semantics and using anonymized data. The methods presented are focusing on continuous-scale numerical data.

The research in [13] presents a methodology based on good practices to offer privacy of medical data, in [14] is presented a platform which offers privacy protection on personal health record.

A natural step in electronic medical data management is to introduce cloud computing technologies in the process. The cloud can solve problems related to the storage, access and processing for medical data, all of this done in a secure way. The introduction of cloud as a solution for medical data storage and retrieval das been discussed and validated in literature, for example in [15] cloud computing is used to share personal health records, [16] presents how cloud can be used to implement a management system for electronic medical records.

The problems related to the integration of cloud computing in medical data processing has been discussed in [17], [18] and [19], while problems related to patient data privacy in cloud have been discussed in [20].

The paper is presenting an information system which is using mobile and cloud services in order to help physicians to manage the observations in the patient observation sheet, allowing manipulation of medical data in a secure way.

\section{The Information System}

The system which will be described next is an information system which allow the user to acquire medical observation data, to store, process and retrieve information from the patient observation sheet. 


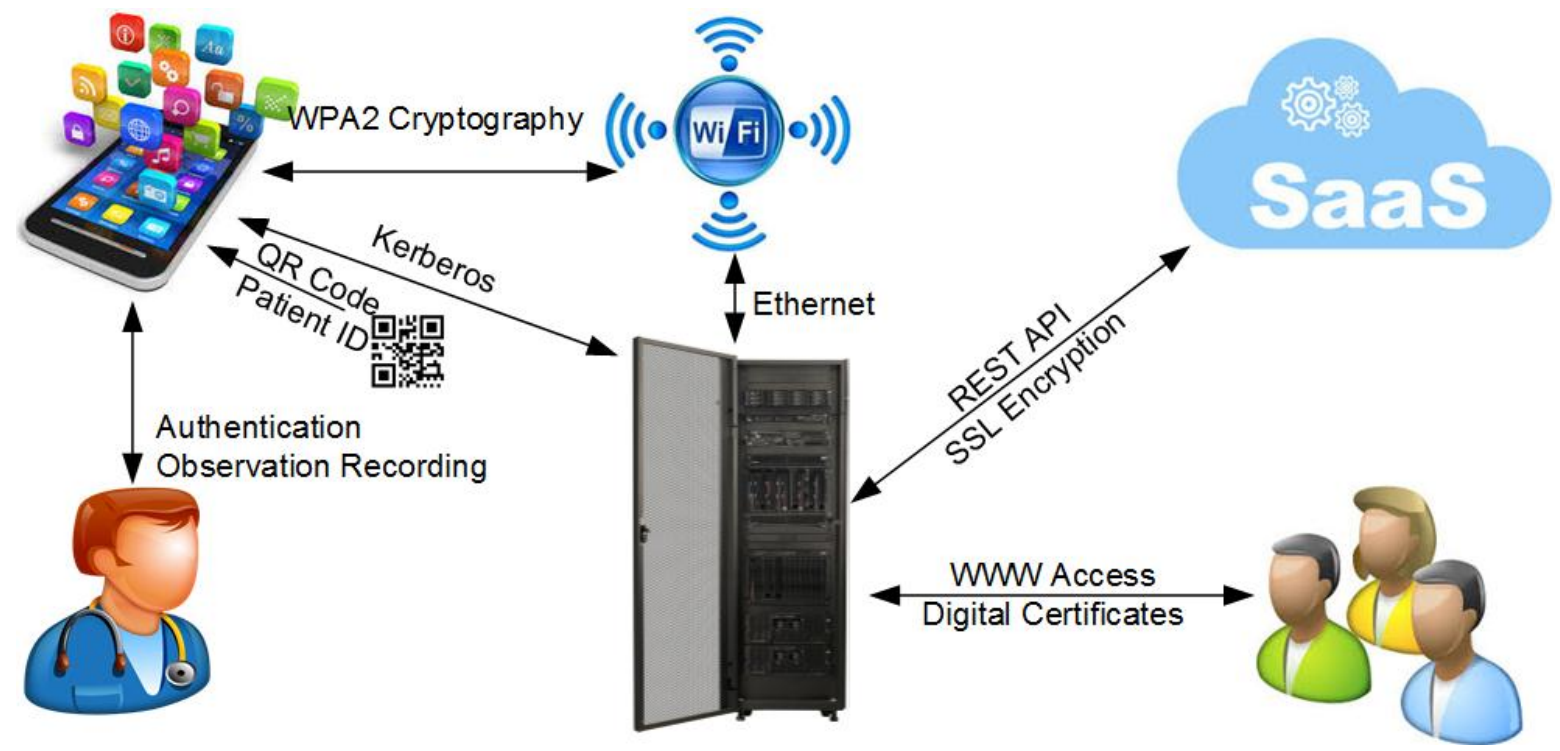

Figure 1. The information system.

The main function of the system is to allow the physician to fill the observation data into the patient clinical observation sheet rapidly, accurate and without omissions, and this without the help of another person, in this way the system is allowing the physician to use his time more effective. The approach is to use a smart phone to record the physician observations, the voice is recorded in $\mathrm{mp} 3$ format and the file is sent to a server which sores the file but also is sending the file to be processed by a Speech to Text Software as a Service (SaaS) cloud service which returns the transcript. The transcript is then imported in the patient clinical observation sheet and then printed and attached next to the bed of the patient.

The information system presented in Figure 1, has three main components:

- A mobile application which can be executed on iOS or iOS or Android phones

- A set of tools running in a private cloud (IBM Service Delivery Manager), which offers authentication services for the mobile application, storage for medical data and connectivity services with a Speech to Text SaaS service

\section{- A Speech to Text SaaS service}

The process of using the system is the following: when the physician starts his visits to the patients he will start an application on his phone for observation data recording. The mobile phone should be connected to the hospital WiFI Hot Spots. When the application starts is requiring a username and a password for authentication, the authentication protocol is Kerberos v5 which uses Advanced Encryption Standard (AES) encryption algorithm, the authentication credentials are not stored on the phone. After the logon the physician can start the patient evaluation, then he will take the patient observation sheet attached to the bed of the patient and scans a Quick Response Code (QR Code) which is printed on the patient observation sheet.

The QR Code contains two identification information:

- The patient name which is used by the physician to verify that the observation he will make will be linked to the correct patient
- A patient ID which is used to identify the patient observation sheet in cloud which will receive the recorded observations.

After verifying that the patient has been identified correctly, the physician has two options:

i) to cancel the process and to rescan another $\mathrm{QR}$ code or,

ii) to start recording the observations

When the physician decides to add his observations to the observation sheet he will start the recording and will speak on the phone microphone (or handsfree) as he is usually using the phone in a conversation. The recording can

be canceled if the physician wants to start all over again also can be placed in pause if the user wants that, the pause mode is also selected if the application is placed in background (for example if the phone rings or if another application is started on the phone in foreground). If the recording is in pause, the recording then can be continued after pressing the resume button on the interface. After the observations have been recorded, the physician can press the send button (Figure 2) and then he can continue the visit by consulting the next patient.

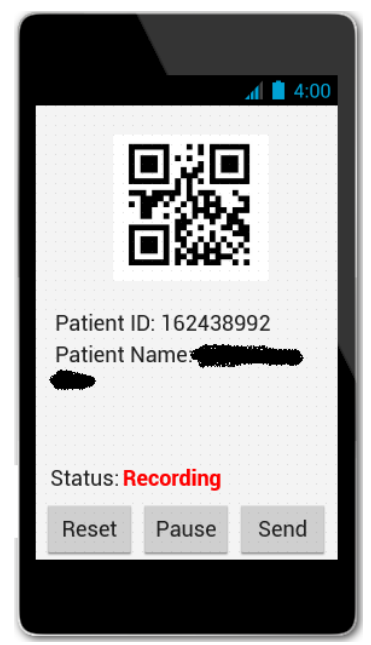

Figure 2. The mobile application. 
When the send button is pressed the recording is converted in $\mathrm{mp} 3$ and is sent into a database on the private cloud which stores the observation sheet, along with the mp3 file other information is stored: the ID of the user which has recorded the observations and the patient ID for which the recording has been done.

After the mp3 file is sent to the database, an agent which is running in cloud takes the file and sends the file to the Speech to Text SaaS service, the file has attached also metadata tagged to it in order to have a correlation with the patient ID. The SaaS service returns the transcript stored into a plain text file or an XML file where each word has also a timestamp identifying the moment in time when the word is pronounced in the mp3 file, in this way corrections on the transcript can be done easily without listening the entire file.

\section{The Mobile Application}

The mobile application is developed in C++ using Embarcadero RAD Studio. The RAD Studio developments environment was used because it offers a set of tools allowing a rapid application development and deployment and also offers the advantage that a single project (application) can be compiled for iOS and Android without changing the code.

The mobile application offers three functions:

- Permits the user to connect to the private cloud services and authenticate through Kerberos protocol

- Allows the physician to identify the patient and patient ID by scanning a QR code

- Allow recording the physician observations in mp3 files and sends those files on cloud for storage

When the application is authenticating is not storing the credentials locally, also after an observation was recorder and transmitted to the cloud the mp3 file is deleted, so on the mobile phone there is no personal information which is kept locally.

\section{Iv. The Private Cloud Services}

The private cloud offers the following services:

1. Allows the physicians to authenticate using the mobile phone and the Kerberos 5 protocol [21], [22].

2. Stores the patient observation sheet data including the physician observations and the transcript obtained after processing the mp3 file without alteration. A versioning system is used which allows to keep history of all changes into the database this feature can be also used in malpractice cases investigation.

3. A SaaS agent is running on the private cloud and monitors the database, when a new mp3 file is uploaded in the database the agent connects to the Speech to Text SaaS service using SSL Encryption, and request that the file to be processed. The request is executed using REST API. After the request is processed and the transcript is returned, the information is placed back in the database.

4. The private cloud offers access to view or edit the patient data based on privileges through a web application. The data which can be viewed or modified includes also the observations transcript which can be corrected, this being a useful feature because the transcript wrongly converts some words which can be corrected later. The web application is using digital certificates, so the connection is encrypted with SSL.

The contains also an mp3 player which can be used to play the mp3 observations files. If the transcript is in $\mathrm{xml}$ format and contains also the timestamps for each word, when the user clicks on a word, the player places the cursor in the mp3 file at the moment when the word is pronounced, and this allows the user to correct the word much easier without playing the entire file.

The services offered by the private cloud are implemented through four distinct applications: i) A Kerberos authentication server; ii) An IBM DB2 Express-C database, which has been selected for the native support of pureXML which offers high performance in managing XML files; iii) a SaaS agent which is continuously monitoring the

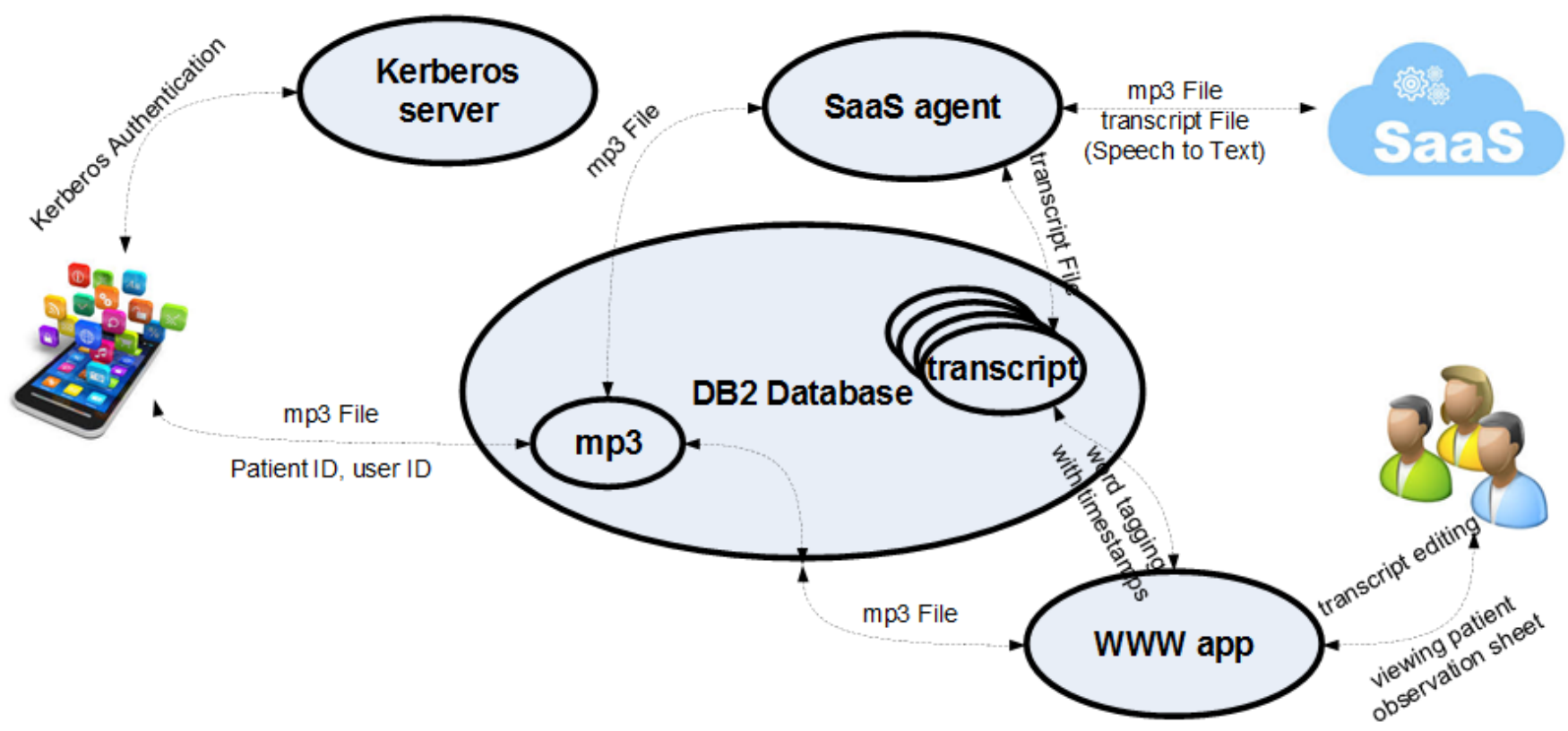

Figure 3. The phone application for observation recording. 
database; iv) a web application for data access.

The connections and the interactions between the applications running in the entire infrastructure can be viewed in Figure 3.

The entire system was designed to have a high level of security, and is using multiple security principles like nested security controls, nonrepudiation services, authentication and authorization controls, least privilege principle, etc.

A special attention has been paid to security and specially to confidentiality of the patient data and the medical data stored and processed by the system. In order to protect the confidentiality multiple security controls have been implemented.

For example, in order to connect to the system, the physician must connect his phone into the private $\mathrm{WiFi}$ network of the hospital. The connection requires the use of WPA2 protocol which is encrypting the data. After connecting to the hospital network, the physician must authenticate using the mobile application, this is done using the Kerberos 5 authentication protocol which is using Advanced Encryption Standard (AES) encryption algorithm for which there is no efficient attack to break the encryption. In this case we have two nested security controls which requires double authentication (at network level and at the application level) and the communication has also two levels of encryption (the data for authentication is encrypted using two algorithms for the connection between the mobile phone and the WiFi access point, and from the access point to the authentication server the data is encrypted through Kerberos). Kerberos is encrypting the data only for authentication and WPA2 protocol uses encryption for all traffic between the mobile phone and the hospital network.

Nonrepudiation mechanism implies that any action executed by a user of the system will not be possible to be denied by the user in cause. This principle has been implemented at the level of the mobile application and at the level of the web application in such way that any change of the observation sheet at the level of the observation data will be saved into a new version of the observation sheet, in other words, a data which was recorded will not be erased, so nonrepudiation will be in effect having all the activity of the user at the level of the observation sheet stored and accessible for audit purposes or even for investigating malpractice cases.

The least privilege principle is used at the web application level and at the database level, where multiple users and roles have been defined:

- The roffice role is used at the hospital reception in order to create the patient file and the observation file, also here a physician can be associated with the patient depending on the speciality and the type of investigation the patient requires.

- The role physician is the only role which can use the mobile application in order to add observations into the patient observation sheet, this role also can be used to add data or modify data on the observation sheet of the patient.

- Other role is guest, this role is associated to specialists which are not working in the hospital, but they are collaborating with the hospital staff, sometimes when a second opinion is required from a specialist, the currant physician can associate the observation sheet with the specialist which can connect remotely, can see the patient observation sheet (which does not contain any personal data) and can only add a comment to the observation sheet which the physician can see and can take into consideration when is changing the medication or is re-evaluating the patient.

- Another role is the role student, which is similar with the role guest, but it has only read only rights, this role is used for training the resident students which can be associated with multiple observation sheets by the physician in order to see how complicated cases have been treated in the hospital.

- There are also other roles like administrator which can add new users associate roles, make backups, etc. Another important role is monitor which allows to see every change in the system, this role being used for audit purposes.

The system is compliant with EU GDPR regulation, and is not sending any patient data outside the hospital, the mobile application is not storing on the phone any data, the SaaS agent is processing only audio information which cannot be used to identify the patient, also the only role which has access to patient data is the roffice role which can use the web application only from the hospital network.

\section{v. Conclusion}

The paper is presenting an information system which is helping the physicians in their daily patient visits to add their observations into the patient observation sheet by using only a smart phone.

The observations in mp3 are sent to a Speech to Text SaaS service (hosted by Google or Vocapia) and the transcript can be corrected later if needed.

Using the observations recorded three tests have been made:

1. The first test was used to process the mp3 files without alteration, this means that the audio file contained also background noise and voices. In this case the recognized word represents $57 \%$ for Vocapia and Google $75 \%$.

2. The second test was conducted on files which have been filtered to reduce the background noise and other voices. The results in this case improved as follows: Vocapia $73 \%$ and Google $84 \%$.

3. The third test has been executed on files where the recordings have been done in a controlled manner: the physician voice constant and without inflexions, and also the other personnel dear the physician kept silence during the recording. The results in this case have been also improved: Vocapia $86 \%$ and Google $93 \%$.

For each test we used $20 \mathrm{mp} 3$ files, the improvement of the results during the three tests was significant, but not all words were recognized (mainly because the medical terms are not in the dictionary), so the physician must spend an additional to correct the transcript. Even if Google service has obtained better results the physician will spend more 
time to correct the file because the transcript is in clear text an is not containing time stamps. On the other hand, the Google service used is free. Vocapia offers the transcript in $\mathrm{xml}$ with timestamps which allows a faster correction but also the ability to add words in the dictionary by improving the service or using locally a machine learning algorithm to train new words.

Regarding the security the system uses local connections which are encrypted using WPA2 protocol for WiFi connections and AES protocol for authentication. Connections in Internet (accessing the SaaS service) are encrypted with SSL and no personal information is sent outside the hospital. The connections to the web applications are also encrypted using digital certificates.

\section{Acknowledgment}

This work was supported by a grant of the Romanian National Authority for Scientific Research and Innovation, CNCS/CCCDI - UEFISCDI, project number PN-III-P2-2.1PED-2016-1336, within PNCDI III.

\section{References}

[1] K. Fujimotoa, T. Nagai, S. Okazaki, M. Kawajiri and K. Tomiw, "Development and verification of child observation sheet for 5-yearold children", Brain and Development, vol. 36, Issue 2, pp. 107-115, February 2014.

[2] L.A. Shanley, C. Hronek, M. Hall, E.R. Alpern, E.S. Fieldston, P.D. Hain, S.S. Shah and M.L. Macy, "Structure and Function of Observation Units in Children's Hospitals: A Mixed-Methods Study", Academic Pediatrics, vol. 15, Issue 5, pp. 518-525, SeptemberOctober 2015.

[3] H. Wang, Q. Wu, B. Qin and J. Domingo-Ferrer, "FRR: Fair remote retrieval of outsourced private medical records in electronic health networks", Journal of Biomedical Informatics, vol. 50, pp 226-233, August 2014.

[4] J. Li, C. Liu, B. Liu, R. Mao, Y. Wang, S. Chen, J.J. Yang, H. Pan and Q. Wang, "Diversity-aware retrieval of medical records", Computers in Industry, vol. 69, pp. 81-91, May 2015.

[5] R. Chauhan and A. Kumar, "Cloud computing for improved healthcare: Techniques, potential and challenges", E-Health and Bioengineering Conference (EHB) 21-23 Nov. 2013, pp. 1-4, 2013.

[6] K. Rosenbeck Gřeg, R. Chen, A. Randorff Hřjen and P. Elberg, "Content analysis of physical examination templates in electronic health records using SNOMED CT", International Journal of Medical Informatics, vol. 83, Issue 10, pp. 736-749, October 2014.

[7] J.T. Carter, "Electronic Medical Records and Quality Improvement", Neurosurgery Clinics of North America, vol. 26, Issue 2, pp. 245-251, April 2015 .

[8] O. Stan, D. Sauciuc and L. Miclea, "Medical informatics system for Romanian healthcare system", E-Health and Bioengineering Conference (EHB) 24-26 Nov. 2011, pp. 1-4, 2011.

[9] J.L. Fernández-Alemán, I.C. Seńor, P.Á. Oliver Lozoya and A. Toval, "Security and privacy in electronic health records: A systematic literature review", Journal of Biomedical Informatics, vol. 46, Issue 3, pp. 541-562, June 2013.

[10] A. Gkoulalas-Divanis, G. Loukides and J. Sun, "Publishing data from electronic health records while preserving privacy: A survey of algorithms", Journal of Biomedical Informatics, vol. 50, pp. 4-19, August 2014.

[11] G. Perera, A. Holbrook, L. Thabane, G. Foster and D.J. Willison, "Views on health information sharing and privacy from primary care practices using electronic medical records", International Journal of Medical Informatics, vol. 80, Issue 2, pp. 94-101, February 2011.

[12] S. Martínez, D. Sánchez and A. Valls, "A semantic framework to protect the privacy of electronic health records with non-numerical attributes", Journal of Biomedical Informatics, vol. 46, Issue 2, pp. 294-303, April 2013.
[13] C. Riou, J. Fresson, J.L. Serre, P. Avillach, L. Leneveut and C. Quantin, "Guide to good practices to ensure privacy protection in secondary use of medical records", Revue d'Épidémiologie et de Santé Publique, vol. 62, Issue 3, pp. 207-214, June 2014.

[14] P. Van Gorp, M. Comuzzi, A. Jahnen, U. Kaymak and B. Middleton, "An open platform for personal health record apps with platform-level privacy protection", Computers in Biology and Medicine, vol. 51, pp. 14-23, 1 August 2014

[15] J. Liu, X. Huang and J.K. Liu, "Secure sharing of Personal Health Records in cloud computing: Ciphertext-Policy Attribute-Based Signcryption", Future Generation Computer Systems, vol. 52, pp. 6776, November 2015.

[16] J. Haskew, G. Rř, K. Saito, K. Turner, G. Odhiambo, A. Wamae, S. Sharif and T. Sugishita, "Implementation of a cloud-based electronic medical record for maternal and child health in rural Kenya", International Journal of Medical Informatics, vol. 84, Issue 5, pp. 349-354, May 2015.

[17] P.J. Hsieh, "Healthcare professionals' use of health clouds: Integrating technology acceptance and status quo bias perspectives", International Journal of Medical Informatics, vol. 84, Issue 7, pp. 512-523, July 2015.

[18] P. Dhivya, S. Roobini and A. Sindhuja, "Symptoms Based Treatment Based on Personal Health Record Using Cloud Computing", Procedia Computer Science, vol. 47, pp. 22-29, 2015.

[19] W. Lin, W. Dou, Z. Zhou and C. Liu, "A cloud-based framework for Home-diagnosis service over big medical data", Journal of Systems and Software, vol. 102, pp. 192-206, April 2015.

[20] J.J. Yang, J.Q. Li and Y. Niu, "A hybrid solution for privacy preserving medical data sharing in the cloud environment", Future Generation Computer Systems, vol. 43-44, pp. 74-86, February 2015.

[21] C. Todd and N. L. Johnson, Jr., "Chapter 3 - Kerberos Server Authentication", Hack Proofing Windows 2000 Server, pp. 63-104, 2001.

[22] J. De Clercq and G. Grillenmeier, "6 - Kerberos", Microsoft Windows Security Fundamentals, pp.303-408, 2007

\section{About Author (s)}

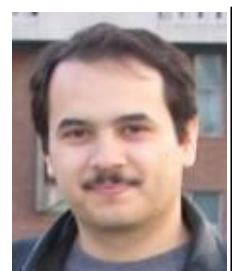

Florin ANTON $(\mathrm{PhD})$ is currently associate professor at University Politehnica of Bucharest. His scientific interests include: high performance equipments, computer security, robotics and artificial vision.

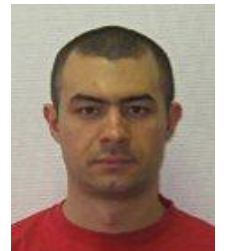

Silviu RAILEANU $(\mathrm{PhD})$ is currently associate professor at the University Politehnica Bucharest. His scientific interests include: agent orientation in industry and services and distributed intelligence for automation systems.

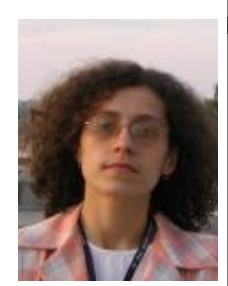

Silvia ANTON $(\mathrm{PhD})$ is lecturer at University Politehnica of Bucharest, Faculty of Automatic Control and Computers. Her scientific interests include: integration of robots in manufacturing and digital logic systems.

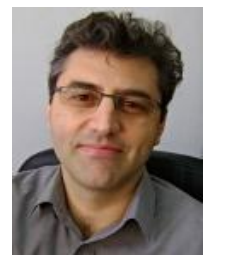

Associate Prof. Nick Andrei IVANESCU develop activities of scientific and applied research in the field of industrial process control using PLCs and microcontrollers, data acquisition and processing, and discrete event systems. 